\title{
EL SIGNIFICADO DEL TRABAJO HOY
}

\author{
Juan José Castillo
}

Universidad Complutense de Madrid

\begin{abstract}
RESUMEN
Para enmarcar y dar sentido a la plétora de libros y artículos que analizan el significado del trabajo, y tomando como pretexto algunas importantes publicaciones norteamericanas, se propone una periodización de las distintas fases que ha atravesado la producción sociológica sobre la crisis del valor trabajo, insertándola en su contexto social, y propiciando así una reflexión que apunta en la dirección de una sociología de la sociología.
\end{abstract}

Permítanme que, como marco de mi argumentación, en lo que sigue, les cuente antes una reciente experiencia personal. A comienzos de noviembre de 1995 llegué a París para una estancia de seis meses. Mi objetivo allí era, fundamentalmente, el dar forma final a un informe de investigación, dentro de un proyecto internacional, sobre la eventual emergencia de nuevos modelos productivos en Europa y en España, particularmente. Hace años que, por formación y vocación, sigo con mucha atención y proximidad la producción francesa en ciencias sociales del trabajo, pero, en esta ocasión, debía además documentarme sobre el debate tan vivo y abundante sobre la crisis del trabajo, las nuevas formas de empleo y actividad, las políticas de empleo, la precariedad creciente de los empleos y las respuestas sindicales ante esa situación. Por ello revisé con minuciosidad y detalle las distintas

\section{Reis}


escuelas y posiciones, aunque ahora no pueda dedicarles el tiempo y comentario que se merecen.

Me encontré con una verdadera plétora de trabajos, libros, investigaciones y números especiales de revistas científicas que no hacían sino traslucir un clima de actualidad del trabajo mayor que nunca, quizá en los últimos diez años: ya fuera para predicar que es un valor en trance de desaparición (Méda, 1995) o para hablar de él en el negativo y reducido marco del crecimiento del paro.

Más aún, el 5 de octubre de 1995 se había inaugurado un ciclo de conferencias-debates públicos (una muestra más de la actualidad del asunto) en el Centro Pompidou, bajo el título genérico "Les mutations du travail». La conferencia inaugural estuvo a cargo de Alain Touraine, quien no sabemos si apoyó la idea de la desaparición del trabajo en su intervención, titulada "Au délà de la société du travail?».

El campo intelectual relacionado con la sociología del trabajo, del empleo, de las políticas sociales o de la crisis del Estado de Bienestar, que comprende en Francia, sólo en sociología, un gran número de laboratorios, trata, obviamente, con enfoques distintos lo que no se duda en señalar como el problema, o uno de los problemas, mayores con que se enfrentan las naciones europeas, y, como es bien sabido, no sólo ellas. Sin embargo, si hubiera de identificarse, en ese otoño de 1995, una corriente dominante, ésta hubiera podido caracterizarse por querer situar al trabajo fuera del centro de los valores y de la constitución de la sociedad.

Todavía el 23 de noviembre podía tener lugar el primero de los debates anunciados, que se proponía una tarea mayúscula como objetivo: «Refonder la notion de travail». Allí los profesionales de las ciencias sociales especulaban, en un clima social ya caliente, sobre el «trabajo que abandona la sociedad postindustrial»; sobre si en verdad las francesas y los franceses no tienen al trabajo como centro de sus vidas, sobre el escaso peso que tiene en la conformación de la personalidad, y sobre lo poco dispuestas que están hoy las personas, ni siquiera quienes tienen un trabajo de los que antes se llamaban "normales»: con contrato de duración indefinida, seguridad social, expectativas de carrera aunque fueran mínimas..., a implicarse en acciones colectivas en defensa de esos valores, y menos aún de forma solidaria.

Pues bien, la sesión siguiente del seminario, dos semanas después, ya no pudo tener lugar: toda Francia, y especialmente París, estaba «en huelgas», como se decía ya popularmente. Y esas huelgas, de trabajadores públicos, con contratos de por vida, eran aplaudidas y seguidas por los contratados precarios, por los temporales, por aquellos que, según los cánones de los investigadores, debian enfrentarse a los huelguistas. Esas huelgas mostraban en los cheminots una concepción del trabajo que comprende todo lo largo de la vida, como colectivo con historia, que chocaba de frente con las apreciaciones de los expertos. Esas huelgas mostraron la capacidad de que un colectivo reducido pudiera defender reivindicaiones que despertaban la solidaridad de quienes no 
tenían la posibilidad de luchar por cuestiones semejantes, aunque las sintieran como algo necesario.

Los mismos sociólogos que se aprestaban a intervenir en los debates sobre el fin del trabajo, desde sus laboratorios y despachos, no comprendían nada de lo que pasaba. Todas sus predicciones chocaban con una realidad tercamente solidaria. Ellos daban explicaciones que, para quien ha seguido de cerca los "movimientos sociales», como se les llamaba oficialmente, mostraban más bien su impotencia e incapacidad de dar una respuesta científicamente satisfactoria: empezaron a hablar de "arcaísmo», de huelga "corporativa», de reivindicaciones trasnochadas. Todo con tal de no reconocer que la realidad a veces tiene razones (y no digamos sentimientos y significados...) que el sociólogo o economista ignora.

Estos hechos tienen para nosotros una importancia fundamental. Primero, porque nos muestran, en conjunto, un esquema crítico, global, de nuestra propia situación como científicos en la sociedad. Comienzan ya a publicarse algunos análisis apresurados de aquellos acontecimientos (Futur Antérieur, 1996), pero lo que me parece más importante de estos hechos es que nos estimulan (o nos obligan) a reflexionar sobre nuestros propios marcos de análisis: ¿qué problemas de investigación, y cómo, debe construir la sociología del trabajo para poder abordar la situación que muestran las huelgas de noviembre y diciembre de 1995 en Francia, para ser capaces de dar explicaciones satisfactorias?

En segundo lugar, y para lo que aquí nos interesa inmediatamente, el significado del trabajo, ese conjunto de hechos nos obliga a plantearnos en qué medida la producción científica corre paralela a la preocupación social, y la refleja en alguna medida, o bien, como este importante acontecimiento nos revela, circula por otro lugar, que parece relativamente autónomo. Casi, literalmente, desvaría. En todo caso, nos ayuda a mantener viva la necesidad de insertar, aunque ello sea mínimamente, las producciones científicas en su contexto.

\section{EL SIGNIFICADO DEL TRABAJO EN SU CONTEXTO}

La crisis del valor trabajo, o el cambio en las actitudes ante el trabajo, como se le solía llamar antaño en Europa, ha conocido en los últimos veinticinco años distintos altibajos como protagonista de la producción científica en las ciencias sociales (Accornero, 1980). De los años de protagonismo de finales de los setenta, a su práctica desaparición de la agenda de investigación a mediados de los ochenta, por poner dos ejemplos contrapuestos.

La primera gran oleada de investigaciones y publicaciones en torno a la crisis del trabajo, entonces bajo la polémica denominación de «degradación del trabajo", está directamente relacionada con la contestación obrera en fábricas y 
oficinas, especialmente agudizada en la "gran metrópoli proletaria» (Turín, Detroit, Madrid...), que tuvo en los años 1968-1972 su gran ciclo delle lotte, como acuñó con acierto Alessandro Pizzorno.

El movimiento obrero, la crítica a la organización capitalista, en los propios procesos productivos, está en la raíz de tantos y tantos estudios que se sucedieron desde 1974, sobre "nuevas formas de organización del trabajo", sobre mejora de las condiciones de trabajo, sobre las actitudes ante el trabajo. Uno de los objetivos fundamentales era intentar devolver a las trabajadoras y trabajadores un trabajo con contenido o significación, recomponer un trabajo cuyo despiece, cuya fragmentación y división, le había llevado a ser la causa principal de explicación de la pérdida de interés por el trabajo, del «frenado» (una vez más), y hasta del sabotaje o la falta de colaboración: conflictos que estaban entonces al orden del día con una excesiva regularidad... para el gusto de los empresarios.

El caso es que, con la distancia que nos dan los años transcurridos, se puede afirmar ahora que la Sociología del Trabajo renovó entonces su equipamiento conceptual, sus herramientas y, desde luego, sus problemas de investigación, espoleada por lo que los «años de las luchas» condensaron en capacidad de influencia social, y no sólo en los centros de trabajo, de los trabajadores y sus sindicatos. Trabajo y capital monopolista, de Harry Braverman, es una buena muestra de esas nuevas preocupaciones y planteamientos.

Desde entonces, y casi como un ciclo de diez años, los centros de atención de la mainstream de las ciencias sociales del trabajo se han focalizado en temas o argumentos que han prevalecido en cada década: los «nuevos conceptos de producción", emblemáticamente representados por ¿El fin de la división del trabajo?, de Horst Kern y Michael Schumann, en 1984; y junto a él otros dos libros, muy distintos pero complementarios, Divisions of labour, de Ray Pahl; y The second industrial divide, de Michael Piore y Charles Sabel. Y, en los noventa, la lean production, como contrapunto y "final de la historia» de la organización del trabajo, como generalización del «modelo japonés» (veáse el monográfico de Sociología del Trabajo, núm. 27, junio 1996, «La emergencia de nuevos modelos productivos»).

Al final de los años setenta y principios de los ochenta tenemos, por tanto, una primera etapa de gran producción de artículos y libros sobre la que se denominó doble crisis del trabajo: crisis de cantidad, esto es, pérdida de empleos; y, la que se consideraba entonces más importante, crisis de calidad del trabajo, y con ella de su significado social, al igual que para las personas. Esos estudios, y más de un libro, llegaron a afirmar que nos hallábamos, y especialmente cuando se trataba de los jóvenes, ante una «alergia al trabajo». Y se crearon instituciones nacionales y europeas (por ejemplo, la European Foundation for the Improvement of Living and Working Conditions, 1975) dedicadas a "mejorar las condiciones de trabajo», como paso primero e imprescindible para que ese trabajo volviera a tener sentido para los trabajadores, para que volvieran a implicarse en el mismo (un estado de la cuestión, en Castillo, 1987). 
Pero la historia, y las estrategias empresariales, se mueven de forma compleja y (tan sólo) aparentemente contradictoria. Aquel movimiento, que en otros lugares se denominó de la calidad de la vida de trabajo (Quality of working Life), para devolver a los trabajadores el gusto por el trabajo bien hecho, como dijo un presidente del gobierno español en 1982, se vio acompañado (o minado subterráneamente) por un conjunto de cambios de estrategias, detectables ya también a mediados de los años setenta, y que iban a terminar por devolver el amor al trabajo a los trabajadores a base de hacerlo escaso y precario.

La primera línea de transformación radical del tejido productivo, que hoy vivimos ya como algo establecido y «normal», se llamó primero decentramento produttivo, fragmentación empresarial que creaba o fomentaba la creación de un tejido a base de microcentros de trabajo. La apariencia hizo que esos años ochenta se acabaran llamando los años de las pequeñas empresas. Con formas diversas de relaciones entre ellas, y de subordinación a empresas grandes o medianas, que siguen concentrado en ellas el poder de decisión sobre las formas que adopta la "división del trabajo entre empresas», sobre salarios y beneficios.

Los estudios sobre la reorganización de la gran empresa se abordaron, en esos mismos años ochenta, desde la perspectiva, casi única, de las «nuevas tecnologías»; ese discurso avasallador e impuesto desde fuera del campo disciplinario allanó, literalmente, los problemas planteados sobre el significado del trabajo, remitiéndose a los viejos y caducos esquemas del determinismo tecnológico: las consecuencias sociales de las nuevas tecnologías eran inevitables, aunque se pudieran proponer "paños calientes" que atemperaran lo que los expertos del sindicato CFDT llamaron los «estragos del progreso».

El significado del trabajo quedaba reducido a apéndice menor en este movimiento que minusvaloraba cualquier forma de implicación de los trabajadores, por inútil o imposible. Sólo la investigación de excelencia no se dejó deslumbrar por la superficie plateada de tanto informe trivial como se produjo, al menos en Europa, en esos años: y así comenzaba a descubrirse que los sistemas complejos de producción, por ejemplo los sistemas flexibles de fabricación (SFF), necesitaban, más que los sistemas tradicionales, de obreros muy implicados en la producción y con unas calificaciones generales, de un nivel superior y distinto (Castillo, 1991). Se dejaba la puerta abierta a lo que va ser una pieza del discurso central de los años noventa que predica, paradójicamente, unos trabajadores fluidos, para los que la empresa no tiene ninguna atadura, a la vez que los quiere implicados e interesados en su trabajo (Linhart, 1991).

Por lo demás, mediados los años ochenta, y agotado el impulso del «ciclo de las luchas», toma el relevo de aquella preocupación por el valor trabajo la creciente asunción de que los problemas que han de estudiarse ahora, en cuanto al significado y vivencias del trabajo, están vinculados a la desaparición del trabajo y, mientras esto acaba de suceder, a su precarización masiva. Todo ello sobre un conjunto tramado de transformaciones que han llevado a una situa- 
ción novedosa entonces, la fluidificación de los sistemas productivos; una situación que hoy, encaminando el fin de los noventa, está ampliamente consolidada (Castillo, 1994; Aronowitz y Di Fazio, 1994).

\section{2. ¿QUÉ HAY DE NUEVO EN LA LITERATURA ACTUAL?}

El libro editado por Frederick Gamst, Meanings of work, publicado en esta última etapa, y situado, como el propio autor indica en varias ocasiones, en Canadá-Estados Unidos, cumple con esa voluntad de integrar las distintas etapas y aproximaciones que se han ido acumulando a lo largo de los años.

Lo primero que hay que agradecerle a Gamst es el recuperar y continuar una tradición de investigación y trabajo de campo común a la antropología y la sociología, que le lleva a preguntarse cómo se justifica la misma separación de los Departamentos universitarios encargados de cada una de esas disciplinas (p. xii). Él mismo recupera y revisa más adelante la tradición de estudios norteamericanos, que clasifica y ordena como «The study of the community and its work» (pp. 14-19). Una buena manera de demostrar en la práctica que volver a los clásicos, a veces tan próximos, es la mejor manera de defender una interdisciplinariedad tan alabada por todos como poco practicada y, en el caso de Gamst, una renovación de la antropología que sólo es posible haciéndola, en la práctica: «Anthropology is what the anthropologist does».

El caso es que esta misma voluntad de recuperar una tradición metodológica, en la investigación sobre el trabajo y sus significados, es muy compartida en Europa, hasta el punto de que también la escuela norteamericana de los años veinte a cincuenta se relee, reinterpreta y reintegra en la mejor investigación, como hemos mostrado en un texto reciente (Castillo, 1996; ver Peneff, 1995; Chapoulie, 1984; Platt, 1992; Junker, 1962).

Hay, pues, en este punto gran coincidencia sobre la necesidad de esfuerzos metodológicos que superen el abordaje trivializado del significado del trabajo, que ha producido tanta construcción de hechos que no eran sino el reflejo de las limitaciones, tanto de los esquemas teóricos con que se abordaban como de los instrumentos de recogida de la información, que, fácilmente, reproducían las idées reçues del investigador (Castillo, 1987).

El segundo aspecto - directamente vinculado con el anterior- que da un tono renovado a estos escritos reunidos por Gamst es, en su propia presentación, la vinculación de la formación y el campo como antropólogos, con el trabajo de investigación directo y la preocupación por los problemas más actuales planteados a la sociología del trabajo. Tal y como concluye Goldsmith, uno de los autores reunidos por Gamst, la antropología nos sirve de distanciamiento de nuestra cotidiana y ajetreada familiaridad con el objeto de estudio, el trabajo: sirve para ayudarnos a ver más claro en nuestras propias circunstancias: «we are really not so different from the Mbuti or the Korean diving women", que él ha analizado (p. 86). 
Por citar un ejemplo de Gamst por todos, que, luego, en un segundo texto, él mismo intentará aplicar a los railroaders, que tan bien conoce, tomemos el de la participación paradójica, a la que antes aludíamos: «al asalariado se le supone que debe ser leal a la empresa, pero el empresario puede, y lo hace, liquidar la relación de empleo cuando quiera» (p. 27).

El tercer punto a destacar en este abordaje es el hecho de situar, como decíamos al final del apartado anterior, en el marco de la «degradación» del empleo y de las relaciones laborales, también en los Estados Unidos. En un apartado titulado "The future of work in North America» (pp. 26 y ss.) se encuadra muy gráficamente la pesadilla que viene, según este autor. Que no puede por menos que compararse con la situación en España y Europa, para ver que vamos hacia una (desgraciada) convergencia: el siglo XXI «será la era del trabajador desenraizado, disponible» (p. 28); "una pesadilla global del trabajo» (p. 30). Un marco, por tanto, de interpretación de lo que hoy significa el trabajo muy semejante al panorama descrito por Aronowitz y Di Fazio (1994) en el primer capítulo de su Jobless future: «nuestro argumento - p. xii- es que la tendencia de la actual vida económica global es hacia el trabajador infrapagado o no pagado".

Del propio Gamst, que «orquesta» el libro, se puede, por tanto, alabar su visión de conjunto, que incluye propuestas conceptuales sobre el trabajo, o sobre lo que llama web of rules, artefacto conceptual y descriptivo que permite atravesar los distintos sistemas de relaciones sociales, e interpretarlos. Ahora bien, aquí mismo es donde se echa de menos una mirada a la literatura europea: por ejemplo, su conceptuación del trabajo no está muy lejos de la formulada por Freyssenet (1994) y del riquísimo elenco de reflexiones reunidas en Enigmes, 1994. Su web of rules, "a network of formal and informal regulations of all kinds including practices and related ideology guiding the social relations of all work» (p. 148), cuya formulación inserta en los clásicos que la formularon hubiera ganado mucho con el contraste con obras como Les règles $d u$ jeu, de Jean Daniel Reynaud (1989, 1995), o con la floreciente économie des conventions (veáse, para una relación de obras y autores, Dosse, 1995), o el libro de Salais y Storper (1993) Les mondes de production.

Las demás contribuciones reunidas en este libro son muy irregulares, y no dejan de resentirse de ser en más de un caso ponencias escritas para una reunión científica que tuvo lugar en 1990. Textos como los de Appelbaum sobre "The concept of work in western thought», siendo impecable en su presentación, añaden poco a lo que ya sabíamos, aunque en el libro, pensado también como un textbook, al mismo tiempo que como un libro de frontera en las propuestas de investigación, es un capítulo imprescindible para contextualizar el trabajo y sus imágenes.

La falta de comunicación entre comunidades científicas muy emparentadas se nota también en el buen trabajo de Marietta Baba, "Work and technology in modern industry: the creative frontier» (pp. 120-146). Primero, porque su revisión de los paradigmas establecidos para la interpretación de la relación tra- 
bajo-tecnología es demasiado huérfana de información sobre la inmensa producción, no sólo europea sino también norteamericana, sobre el asunto (veáse, para un panorama, Tertre y Santilli, 1995). Su cultural construction of technology hubiera ganado mucho con los enfoques de la ergonomía de lengua francesa y con el contraste de los métodos ergonómicos, que no difieren mucho de los etnográficos. Más bien se juntan (Castillo, 1989). Según Baba, el trabajo, el trabajador o trabajadora, crea también la tecnología, en su aplicación, modificándola o completándola (p. 136). Buena parte del argumento sustantivo que hace también al trabajo creador de tecnología, precisamente mientras trabaja, está fundado en una constancia: "very complex production technologies rarely works as planned under real operating conditions» (p. 140). Ése es, precisamente, uno de los puntos de partida de ese tipo de aproximación, muy bien ilustrado por De Terssac (1992).

En el capítulo de June Nash, «Post-industrialism, post-fordism, and the crisis in world capitalism», volvemos a estar ante un caso —excelente- de texto de "manual», o sea, de libro de texto, donde se revisan, más para uso de estudiantes que para discutir con la última investigación, una serie de interpretaciones típicas de nuestra disciplina. Pero eso sólo concierne a la introducción del texto. A continuación se aporta un magnífico estudio de caso (pp. 196211) sobre General Electric en Pittsfield. Y aquí volvemos a estar en la punta de la mejor investigación.

Otros capítulos del libro tienen difícil encaje en el conjunto, como el de Judith Blau sobre middle classes, que, siendo muy interesante, sólo puede tener sentido aquí por hablar de la importancia de la cultura en la constitución de estas clases. El texto de Ivar Berg presenta una bien ordenada clasificación de distintos tipos de trabajo que caracteriza como macro, de carácter general, de alcance medio [middle range] y micro, o cuasi plant sociology. Pero este orden debe estar muy condicionado por la específica problemática y discusión en los Estados Unidos, y uno puede sólo beneficiarse de un razonamiento que evidencia los límites del conocimiento en cada uno de los niveles: está bien claro hace tiempo que los estudios generales apenas pueden estar seguros de las tendencias, sin la profundidad, el matiz y la complejidad de los estudios directos sobre el terreno. Pero, en realidad, ese tipo de estudios encarna mucho más en una crítica clásica a la división del trabajo académico: los economistas hacen (o hacían...) sólo estudios generales, sobre tendencias del mercado de trabajo, la mayoría de las veces basándose en datos secundarios; los sociólogos entran en las fábricas, conocen de primera mano (o debieran...) las vivencias del trabajo.

$\mathrm{Ni}$ que decir tiene que ésta es una visión esquemática y falsa: no basta tampoco con clasificar a los investigadores en "naturalistas» o "racionalistas», según tiendan más a ser macro-meso o micro-meso: según la mirada, la complejidad y la capacidad de insertar unos análisis en otros. Se debe observar que en todo este juego de combinaciones no se cita siquiera un título del ya largo debate en las ciencias sociales sobre The macro-micro link, por mencionar un libro muy difundido. 
La originalidad de este tipo de textos — que los hacen poco útiles_ podría estar en una especie de solipsismo, de encerrarse con sus propios problemas, como si, al igual que los viejos macroeconomistas, les costara salir de su cuarto de estar disciplinario. $\mathrm{Y}$, a veces, hasta asomarse a la ventana.

En fin, el libro se cierra con un texto más programático que analítico de Amitai Etzioni, al que hay que agradecer que recuerde el aspecto de proyecto que toda obra humana tiene. El sentido del trabajo, su significado, tiene en cada persona, según su propia historia, su experiencia, sus expectativas, su clase social, un despliegue particular. Si a ello le añadimos los variables contextos sociales, ya puede identificarse un panorama complejo.

Research in the Sociology of Work es una publicación anual que comenzó su andadura en 1981. Sus editores son Richard Simpson e Ida Simpson. A esta última le debemos una reflexión y un debate, publicado en Social Forces, que converge con nuestras propias reflexiones aquí, en Europa, y en América Latina: tanto el monográfico de Sociologie du Travail, "Les énigmes du travail», 1994, como mis "¿A dónde va la Sociología del Trabajo?» y «A la búsqueda del trabajo perdido, y de una sociología capaz de encontrarlo». Su artículo, de 1987, "Where have all the workers gone in the Sociology of Work?» hizo papel de manifiesto reflexivo de nuestra disciplina.

El objetivo adelantado por los editores de este volumen 5, 1995, de $R e$ search..., "The meaning of work», es de extrema actualidad: se trata de centrarse en las grandes transformaciones del trabajo y de cómo los trabajadores viven la experiencia de esos cambios. El objetivo propuesto para estas reflexiones es «subjective relations between workers and work. What does work mean to workers? What do they want from it? Do they get what they want? How do feelings about work affect life off the job?» (p. xi). Éstas son las preguntas fundamentales dirigidas a los autores, que responden a ellas desde posiciones y preocupaciones muy distintas, y llevando a cabo investigaciones planteadas y realizadas con criterios metodológicos y presupuestos teóricos también diferentes.

Desde quienes intentan verificar el papel del género, la raza o la etnicidad, en la variación de la satisfacción en el trabajo, hasta quienes van más al corazón de las cosas, planteándose las relaciones básicas que estructuran la situación actual: las relaciones de empleo como relaciones de confianza, por ejemplo (Margaret Krecker).

Por ello mismo, el conjunto de doce artículos, valioso en sí mismo, tiene, a mi juicio, trabajos planteados con los viejos esquemas de la satisfacción en el trabajo, muy poco productivos, de nuevo conocimiento, por mucho que se crucen variables, o que se intente llegar a relacionar esa satisfacción incluso con el rendimiento escolar de los hijos de los trabajadores...

Mis preferencias o preocupaciones me han llevado a leer con mucho mayor interés, en cambio, aquellos artículos que intentan abrir nuevas reflexiones, o que las aportan directamente.

Comenzando por el orden inverso de aparición en Research on The Sociology of Work, el artículo de Susan Goodrich Lehmann, "Costs and opportuni- 
ties of marketization: an analysis of Russian employment and unemployment", ofrece, por lo pronto y por lo menos, una información crítica, que puede servir de punto de partida para una evaluación de esa más que dolorosa transición del socialismo al capitalismo.

El artículo más novedoso, importante y, para mí al menos, inductor a la reflexión sobre nuestro propio gremio, el de los investigadores sociales, es, sin duda, el de Tim Turpin y Stephen Hill, «Researchers, cultural boundaries, and organizational change». A primera vista, podría parecer que un texto que analiza el trabajo de los investigadores, sus carreras desde que son «becarios», pero en Australia, podría decirnos poco a los europeos. Nada más lejos de la verdad: se trata de un artículo al que bastaría quitar los nombres propios, obviamente, para describir trayectorias y tipos que nos son familiares, lo mismo en España que en Francia o Italia, por citar tres países que conozco: los bounded pragmatists; the evangelists; the warlords; o the controlled marketeers.

Le sigue en interés el artículo de Margaret L. Krecker, «From the "instinct of workmanship" to "gift exchange": employment contracts, social relations of trust, and the meaning of work». Muy próximo a las distintas reflexiones, tanto europeas como americanas, sobre el trabajo situado, las teorías de las convenciones, el gobierno [governance] de los sistemas productivos y los distritos industriales, el work embedded, etc. Aunque, todo hay que decirlo, planteando bien los problemas, no avanza mucho más allá de donde los ha colocado la investigación hasta el presente.

En «Work orientation, job satisfaction, and ethnicity: a focus group perspective» (pp. 45-85), Lawrence Bobo y sus colegas presentan una discusión introductoria y un planteamiento novedoso para la preparación de una futura investigación, de la que ésta, que podríamos llamar pre-encuesta, proporciona, por el método con el que se ha llevado a cabo, muy inteligentes insights, muy iluminadoras hipótesis.

Y llegamos así al primero de los textos incluidos en este monográfico, el artículo de Jan Karlsson, "The concept of work on the rack». Primero en la revista y último en mi lista de buenos trabajos. Porque, habiendo comenzado con un planteamiento formalmente correcto, acaba con una argumentación que más de una vez parece como si hubiera un salto o corte en su desarrollo.

No acepta Karlsson la "extensión» del concepto de trabajo, confundiendo - a mi juicio- el concepto con la realidad del trabajo. Su crítica parte de enfrentarse a un texto de Arlene Daniels (1987), "Trabajo invisible» (equivalente, más o menos, a los trabajos que tan certeramente despliega, en España, María Angeles Durán o, en Brasil, Alice Abreu). Tanto en Daniels como en Durán, Abreu y otras autoras - especialmente canadienses-, lo que se propone no tiene nada que ver, como Karlsson afirma, con un retorno a un folk concept de trabajo, una extensión que, según él, sería perjudicial para la Sociología del Trabajo. Y sus propuestas de "formas de trabajo" no son tampoco gran innovación, puesto que la identificación de sus cuatro formas es puramente tipológica y oportunista: para intentar servir a un argumento poco claro. El 
texto salta abruptamente de la pequeña muestra del trabajo de investigación del propio Karlsson - muy sugerente, por otro lado, de la fecundidad del método biográfico - para terminar el texto volviendo a una crítica de Daniels que no tiene ya lugar en la propia organización de la discusión del autor.

De hecho, habría que decir que éste ignora antiguos esfuerzos de formalización más logrados, como, por citar tres, los de Bagnasco en Italia (Stato e Mercato, núm. 1, 1981), "La cuestión de la economía informal»; de Ignacy Sachs en Francia en los años ochenta; o el fundamental libro de Ray Pahl, en el Reino Unido, Divisions of labour (1984), traducido al español por el Centro de Publicaciones del Ministerio de Trabajo.

Hay, finalmente, otros artículos que uno no entiende cómo, después de veinticinco años de crítica demoledora, se siguen produciendo. Glenn Firebaugh y Brian Harley estudian las "Trends in job satisfaction in the United States...» (pp. 87-104). Si algo faltaba para descartar de una vez este tipo de investigaciones, la que comentamos podría bien ser su epitafio. Los autores analizan esas tendencias de evolución a lo largo de veinte años, desde 1972, para llegar a conclusiones tan poco estimulantes como ésta: "On average, bluecollars workers and service workers are as satisfied now as in 1972» (p. 102): el tiempo, precisamente, de la desindustrialización de América, o del downsizing de su economía para tomar el título de la conocida serie de artículos de marzo de 1996 en el New York Times, y nada ha cambiado en la percepción de las gentes.

El resultado les parece a los autores intriguing. Que un concepto y sus tecnologías, así elaborado, permanezca congelado a lo largo de décadas les lleva a este comentario: "job satisfaction as conventionally measured, then, appears to be insensitive - or sensitive in unexpected ways - to the reestructuring of American workforce». Y a concluir que hay que seguir investigando... A uno se le ocurre que lo que hay que investigar, haciendo una buena sociología de los sociólogos, es el sistema de ciencia que sigue financiando estos estudios que concluyen que no han descubierto nada, pero hay que seguir investigando.

Y esa misma apertura hacia la sociología de la sociología le asalta a uno leyendo el libro de Catherine Casey, Work, self and society. Porque tenemos delante un libro correcto, académicamente intachable, pero que para el "profesional» de la sociología del trabajo tiene un cierto aire de acumulación «libresca»: queremos decir, tomando como ejemplo los tres primeros capítulos de presentación de la problemática, sobre el trabajo, su transformación y el self, que el critical discourse sobre el trabajo es un artesano tejido de referencias y citas, encajadas unas con otras con precisión, pero construidas como un deber académico, sin pasión ni aparente toma de posición. En el segundo, la transformation of work, nuevamente la acumulación de referencias es tal $-\mathrm{y}$ sin criterio- que se tiene la sensación de que tan sólo parece que se domina un campo, vasto y complejo, y que se cita de oído. Y, finalmente, otro tanto sucede al completarse el tríptico, sobre los discourses of the self. 
Sin embargo, se pierde esa sensación cuando se entra a presentar - y ésta es la parte central y más amplia del libro- el estudio de la cultura de una gran empresa, en los Estados Unidos, tal y como se nos dice en apéndice metodológico, con etnografic methods, u observación participante, larga y detenida de la autora en la empresa.

Leyendo lo conseguido por ella, y las disquisiciones de su último capítulo, "Revivalism, self and solidarity» (pp. 182-197), uno se pregunta si una confrontación inter e intradisciplinaria no habría orientado mejor y más allá de donde las deja la autora sus conclusiones (Dubois, 1996). Pues los vocabulaires of motives, para usar una vieja denominación de Wright Mills, sobre la calidad total, [Total Quality Management] utilizados por la ideología empresarial «is likely to be used as the basis for renewal of management ideology: using the language of teamwork, continous improvement and partnership, it gives senior management legitimacy for centralisation, the promotion of a unitarist view of organisation and the representation of intensification of work, without greater autonomy as "empowerment" (Webb, 1996: 268).

\section{CONCLUYENDO}

¿Qué enseñanzas podemos extraer de la lectura crítica de los libros que comentamos sobre el significado del trabajo? Como se desprende de las líneas anteriores, abordar hoy esta cuestión central para la interpretación del devenir de nuestras sociedades, ya sean "centrales» o "periféricas», requiere que la investigación se plantee un conjunto de problemas que, en modo distinto y con distinto énfasis, se desprenden del comentario de los textos reseñados.

La primera consideración es que no podemos partir de una posición «adanista», como si fuéramos los primeros en preocuparnos por el significado del trabajo. En la práctica, una revisión de nuestros clásicos debe terminar por una auténtica Aufhebung, esto es, por incluir y superar las experiencias y los logros anteriores.

En segundo lugar, y directamente vinculado a la anterior observación, se necesita hacer un esfuerzo tanto disciplinar como interdisciplinar para no descubrir el Mediterráneo: especialmente la consulta de producciones en otros idiomas (y culturas, por tanto). Para el mundo angloparlante [o anglo reader], se trata de no ignorar trabajos, por poner dos ejemplos llamativos y «antiguos», como los de Romagnoli y Sarchielli (1983); o el fundamental texto de Accornero (1980), a la hora de abordar el significado del trabajo. Y no digamos de la abundante literatura reciente, como esa compleja discusión interdisciplinaria de Yves Clot, en Le travail sans l'homme?, 1995, que parafrasea al Albert Camus de L'homme révolté cuando afirma: «un poco de subjetivo nos aleja de lo social, pero mucho nos acerca a ello» (p. 163).

Y la tercera conclusión o aprendizaje provocado por estos trabajos sobre el trabajo es, simplemente, la consecuencia del enfoque desarrollado en los dos 
momentos que proponemos: se trata de construir marcos complejos en la propia fabricación de la problemática, tanto social como sociológica (un buen ejemplo, en Billiard, 1995). Estas problemáticas, ni que decir tiene, van necesariamente acompañadas de innovadores marcos teóricos y metodológicos. A algunos de los trabajos aquí reseñados les vendría bien, como hemos ya dicho, el confrontarse con trabajos como los de Teiger (1994), para la ergonomía francesa; Leite (1993) o Itani (1992), para Brasil; Nieto (1994) o González de la Rocha (1995), para México...

Sólo así, en esta etapa del fin del milenio, podremos conocer, y, lo que es más importante, entender, el papel y el significado del trabajo en su verdadera dimensión y significación, cuando, como todo el mundo acepta, "profondes transformations sont en cours dans le rapport que les sujets sociaux, et surtout les jeunes, entretiennent avec le travail» (Castel, 1995: 449).

\section{REFERENCIAS}

ACCORnero, Aris (1980): Il lavoro come ideologia, Bolonia, Il Mulino, 222 pp.

Aronowitz, Stanley, y Difazio, William (1994): The jobless future. Sci-Tech and the dogma of work, Minneapolis-Londres, University of Minnesota Press, 392 pp.

BILliard, Isabelle (coord.) (1995): Identités, santé, insertion sociale et nouvelles formes d'emploi, París, MIRE (Mission Interministerielle Recherche-Expérimentation)-Groupe de Travail, 313 pp.

Bouvier, Pierre (1995): Socio-Anthropologie du contemporain, París, Galilée, 176 pp.

CASEY, Catherine (1995): Work, self and society. After industrialism, Londres y Nueva York, Routledge, $241 \mathrm{pp}$.

CASTEL, Robert (1995): Les métamorphoses de la question sociale. Une chronique du salariat, París, Fayard, 490 pp.

CASTILlo, Juan José (1987): "Crisis del trabajo y cambios sociales», en REIS, núm. 38, pp. 7-20. [número monográfico, editado por J.J.C.].

- (ed.) (1989): La ergonomía en la introducción de nuevas tecnologias en la empresa. Problemas y soluciones, Madrid, Ministerio de Trabajo [con la colaboración de Jesús Villena].

- (ed.) (1991): La automación y el futuro del trabajo. Diseño del trabajo y cualificación de los tra-

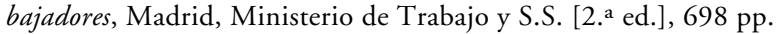

- (1994): El trabajo del sociólogo, Madrid, Universidad Complutense, 427 pp.

- (1996a): "A la búsqueda del trabajo perdido. Y de una sociología capaz de encontrarlo», en A. Pérez-Agote e I. Sánchez de la Yncera (eds.), Complejidad y teoría social, Madrid, Centro de Investigaciones Sociológicas, pp. 73-95. [Una versión notablemente más desarrollada y ampliada se ha publicado en 1997 en Estudios Sociológicos, 1997, México.]

- (1996b): Sociología del Trabajo. Un proyecto docente, Madrid, CIS-Siglo XXI, 208 pp.

- (1996c): «El paradigma perdido de la interdisciplinariedad: volver a los clásicos», primer borrador, julio, $22 \mathrm{pp}$.

Chapoulie, Jean-Michel (1984): «Everett C. Hughes et le développement du travail de terrain en sociologie», en Revue Française de Sociologie, t. XXV, pp. 582-608.

Clot, Yves (1995): Le travail sans l'homme? Pour une psychologie des milieux de travail et de vie, París, Éditions La Découverte, 275 pp.

Cottereau, Alain (1994): «Théories de l'action et notion de travail. Note sur quelques difficultés et quelques perspectives», en Sociologie du Travail, Hors Série, pp. 73-89.

Daniels, Arlene Kaplan (1987): «Invisible work», en Social Problems, vol. 34, núm. 5, diciembre, pp. 403-415. 
Dosse, François (1995): L'empire du sens. L’humanisation des sciences humaines, París, La Découverte, $432 \mathrm{pp}$.

Dubois, Pierre (1996): "Markets in organizations and organizations in Markets», en Work, Employment and Society, vol. 10, núm. 2, junio, pp. 361-376.

ÉNIGMES DU TRAVAIL, LES (1994): número monográfico de Sociologie du Travail, Hors Série, 125 pp.

ERbÈS-SÉguin, Sabine (dir.) (1995): L'emploi: dissonances et défis. Sociologues et économistes en débat, París, Éditions L'Harmattan, 327 pp.

FREYSSENET, Michel (1994): «Los enigmas del trabajo: nuevas pistas para su conceptuación», en Revista de Economía y Sociología del Trabajo, núms. 23-24, pp. 63-71 [monográfico sobre la Sociología del Trabajo en América Latina, editado por Juan José Castillo].

FUTUR ANTÉRIEUR (1996): "Tous ensemble: réflexions sur les luttes de novembre-décembre», monográfico de Futur Antérieur, núms. 33-34, 300 pp.

GaMST, Fredecick C. (ed.) (1995): Meanings of work. Considerations for the Twenty-First century, Nueva York, State University of New York Press, 277 pp.

GONZÁlEZ DE LA RoCHA, Mercedes (1995): «Reestructuración social en dos ciudades metropolitanas: un análisis de grupos domésticos en Guadalajara y Monterrey [México]», en Estudios Sociológicos, vol. XXIII, núm. 38, mayo-agosto, pp. 261-28.

Hughes, Everett Cherrington (1952): "The sociological study of work: an editorial introduction», en American Journal of Sociology, vol. 57, núm. 5, marzo, pp. 423-426 [Special issue: "The sociology of work»].

Junker, Buford H. (1960): Field work. An introduction to the social sciences, Chicago y Londres, The University of Chicago Press, 209 pp. [with an introduction by Everett C. Hughes].

LEITE, Marcia de Paula (1993): "Innovación tecnológica y subjetividad obrera», en Sociología del Trabajo, nueva época, núm. 19, otoño, pp. 3-26.

LinHART, Danièle (1991): "Le travail et la question de sa centralité pour l'univers personnel et social», en Sociologie et Sociétés, vol. XXIII, núm. 2, otoño, pp. 47-49.

MÉDA, Dominique (1995): "La fin de la valeur travail?», en Esprit, núms. 8-9, agosto-septiembre, pp. 75-93 [número cuasi monográfico, "L’avenir du travail»].

NiETo, Raúl (1994): "Antropología del trabajo industrial [aproximación antropológica al conocimiento del trabajo y del mundo industrial en México]», en Sociología del Trabajo, nueva época, núm. 22, otoño, pp. 29-45.

PAHL, R. E. (1988): Divisiones del trabajo, Madrid, Ministerio de Trabajo y S.S. [edición original, Oxford, Blackwell, 1984].

PENEFF, Jean (1996): "Les débuts de l'observation participante ou les premiers sociologues en usine», en Sociologie du Travail, núm. 1, pp. 25-44.

PlatT, Jennifer (1983): "The development of the "participant observation" method in sociology: origin, myth and history", in Journal of the History of the Behavioral Sciences, vol. 19, octubre, pp. 379-393.

- (1992): "Case study" in American methodological thougt", en Current Sociology, vol. 40, núm. 1, primavera, pp. 17-48 [dentro de un número monográfico, "The case method in sociology", editado por Jacques Harmel].

REYNAUD, Jean-Daniel (1989): Les règles du jeu. L'action collective et la régulation sociale, París, Armand Colin [2. ${ }^{\mathrm{a}}$ ed., 1995].

Romagnoli, Guido, y SARChielli, Guido (eds.) (1983): Immagini del lavoro. Una ricerca tra $i$ lavoratori manuali, Bari, De Donato, $292 \mathrm{pp}$.

SALAIS, Robert, y STORPER, Michael (1993): Les mondes de production. Enquête sur l'identité économique de la France, París, Éditions de l'École des Hautes Études en Sciences Sociales, 467 pp.

SimPSON, Richard L., y SIMPSON, Ida Harper (eds.) (1995): The meaning of work. Research in the Sociology of Work, vol. 5, 284 pp.

TeIger, Catherine (1994): "El trabajo, ese oscuro objeto de la ergonomía", en Sociología del Trabajo, nueva época, núm. 22, otoño, pp. 3-28. 
Terssac, Gilbert de (1992): Autonomie dans le travail, París, PUF, 279 pp. [edición en español, Madrid, Ministerio de Trabajo y S.S., 1996].

Tertre, Christian Du, y SANTILLI, Giancarlo (1992): Automatisation et travail: utopies, réalités, débats, des années cinquante aux quatre-vingt-dix, París, PUF, 176 pp.

WebB, Janette (1996): "Vocabularies of motive and the "NEW" management", en Work, Employment and Society, vol. 10, núm. 2, junio, pp. 251-271.

WISNER, Alain (1995): Réflexions sur l'ergonomie (1962-1995), Toulouse, Éditions Octarès, $158 \mathrm{pp}$.

\begin{abstract}
In order to frame and make sense of the plethora of books and papers which analyse the meaning of labour, and using certain important American publications as an excuse, this paper proposes a periodisation of the various phases sociological production has gone through in terms of the crisis of the work value, inserting it in its rightful social context and triggering a reflection which points in the direction of a sociology of sociology.
\end{abstract}

